\title{
A structured summary of a study protocol for a multi-center, randomized controlled trial (RCT) of COVID-19 prevention with Kampo medicines (Integrative Management in Japan for Epidemic Disease by prophylactic study: IMJEDI P1 study)
}

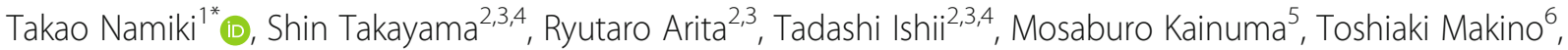
Masaru Mimura ${ }^{7}$, Tetsuhiro Yoshino ${ }^{8}$, Tatsuya Nogami ${ }^{9}$, Makoto Arai ${ }^{9}$, Juichi Sato ${ }^{10}$, Koichiro Tanaka ${ }^{11}$, Hajime Nakae ${ }^{12}$, Hidetoshi Igari ${ }^{13}$, Yoshihito Ozawa ${ }^{14}$, Yuki Shiko ${ }^{14}$, Yohei Kawasaki ${ }^{15}$, Masahiko Nezu ${ }^{1}$ and Takashi Ito ${ }^{16}$

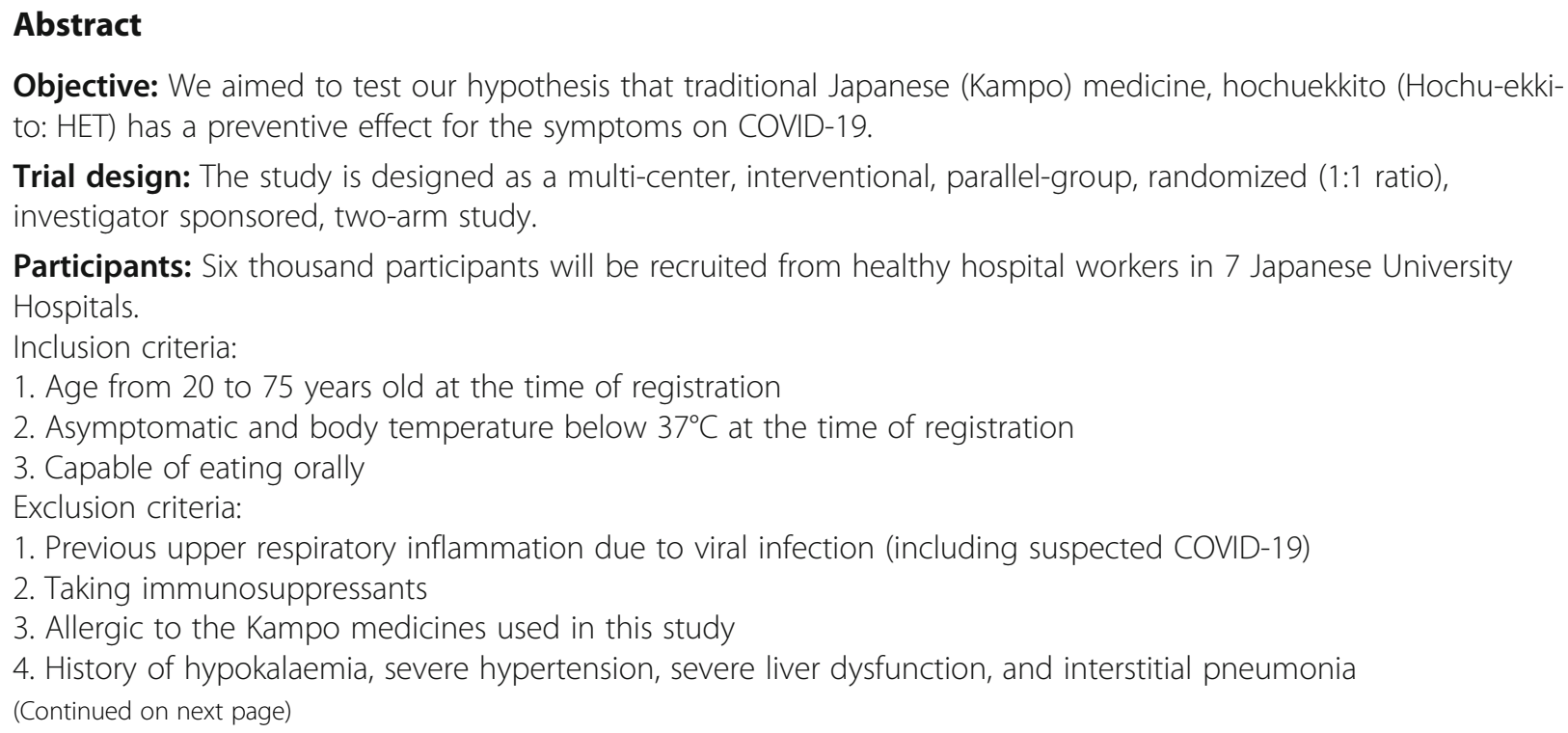

Trial design: The study is designed as a multi-center, interventional, parallel-group, randomized (1:1 ratio), investigator sponsored, two-arm study.

Participants: Six thousand participants will be recruited from healthy hospital workers in 7 Japanese University Hospitals. Inclusion criteria:

1. Age from 20 to 75 years old at the time of registration

2. Asymptomatic and body temperature below $37^{\circ} \mathrm{C}$ at the time of registration

3. Capable of eating orally

Exclusion criteria:

1. Previous upper respiratory inflammation due to viral infection (including suspected COVID-19)

2. Taking immunosuppressants

3. Allergic to the Kampo medicines used in this study

4. History of hypokalaemia, severe hypertension, severe liver dysfunction, and interstitial pneumonia

(Continued on next page)

* Correspondence: tnamiki@faculty.chiba-u.jp

'Department of Japanese-Oriental (Kampo) Medicine, Graduate School of Medicine, Chiba University, 1-8-1, Inohana, Chuo-ku, Chiba 260-8670, Japan

Full list of author information is available at the end of the article

C The Author(s). 2020 Open Access This article is licensed under a Creative Commons Attribution 4.0 International License, which permits use, sharing, adaptation, distribution and reproduction in any medium or format, as long as you give appropriate credit to the original author(s) and the source, provide a link to the Creative Commons licence, and indicate if changes were made. The images or other third party material in this article are included in the article's Creative Commons licence, unless indicated otherwise in a credit line to the material. If material is not included in the article's Creative Commons licence and your intended use is not permitted by statutory regulation or exceeds the permitted use, you will need to obtain permission directly from the copyright holder. To view a copy of this licence, visit http://creativecommons.org/licenses/by/4.0/ The Creative Commons Public Domain Dedication waiver (http://creativecommons.org/publicdomain/zero/1.0/) applies to the data made available in this article, unless otherwise stated in a credit line to the data. 

(Continued from previous page)
5. Regularly taking other Kampo medicines
6. Pregnant or possibly pregnant
7. Participating in other research
8. Judged to be unsuitable for this study by the doctor in charge

Intervention and comparator: Kampo group: participants receive HET in 9 tablets 2 times per day for 8 weeks. Control group: participants receive placebo in the same dosage as the Intervention group - 9 tablets 2 times per day for 8 weeks. Placebo tablets are identical in appearance and package to HET. Taste of placebo is different from that of HET.

The Ohsugi Pharmaceutical Co. Ltd, Osaka, Japan manufactured the placebo and HET.

Main outcomes: Primary outcome: Number of patients with a SARS-CoV-2 RNA by ploymerase chain reaction (PCR) positive result with at least one symptom (fever, cough, sputum, malaise, shortness of breath) during the 12-week study period (including the 4-week observation period after oral administration).

Secondary outcomes:

1. Period from infection to onset

2. Period from the appearance of symptoms to the disappearance of PCR positive

3. Number of days until the appearance or improvement of symptoms

4. Severe stage: presence of hospitalization

5. Shock stage: ICU management required for mechanical ventilation, shock vitals or failure of organ(s) other than lungs

Safety endpoints include numbness in the hands and/or feet, edema, skin rash or other allergic symptoms, and gastric discomfort.

Randomisation: Patients are randomized (1:1 ratio) to each group using minimization implemented with the Electric data capture system (DATATRAK Enterprise Cloud), with balancing of the arms with age range (under 50 years of age or not) and having a history of risk factors for COVID-19 (cardiovascular disease, hypertension, diabetes, respiratory diseases).

Blinding (masking): Only participants will be randomized.

Numbers to be randomised (sample size): The main research hypothesis of this study is that Kampo medicines significantly prevent the onset of COVID-19. It is assumed that the infection rate before the administration of the drug under consideration will be $0 \%$ and that the incidence of COVID-19 thereafter will be 2- 3\%, of which 70\%$80 \%$ will show symptoms of COVID-19. Assuming that the pharmaceutical effect of the drug will be effective in $50 \%$ of patients and that the incidence rates in the placebo and drug groups will be 1.4\%-2.4\% and 0.7\%-1.2\%, respectively, the placebo is calculated at $2 \%$, and the study drug at $1 \%$. Since the frequency of verification is low and the number of cases will be large, we set a total of 10 analyses ( 9 interim analyses and a final analysis). Since the number of cases at the time of the final analysis will be 4,986 under the conditions of $a=0.05$ and a power of $80 \%$ by the Peto method. We set at 600 cases in each interim analysis with an estimated dropout rate of $16.9 \%$. Finally, the total number of cases is set to 6,000 with 3,000 in the placebo group and 3,000 in the HET group.

Trial status: Protocol version 1.3 of October 23rd, 2020. Recruitment start (expected): December $1^{\text {st }}, 2020$. Recruitment finish (expected): December $31^{\text {st }}, 2022$.

Trial registration: This trial is registered in the Japan Registry of Clinical Trials (jRCT) (jRCTs031200150) on 14 October 2020.

Full protocol: The full protocol is attached as an additional file, accessible from the Trials website (Additional file 1). In the interest of expediting dissemination of this material, the familiar formatting has been eliminated; this Letter serves as a summary of the key elements of the full protocol.

Keywords: COVID-19, randomized controlled trial, protocol, Kampo medicines, prophylactic study 


\section{Supplementary Information}

The online version contains supplementary material available at https://doi. org/10.1186/s13063-020-04939-2.

Additional file 1. Full Study Protocol.

\section{Acknowledgements}

The authors would like to thank secretary Ms. Emiko Yoshida for revising the protocol.

\section{Authors' contributions}

T. Namiki, S.T., R.A., T. Ishi, M. K., and T. M. were involved in design of this trial. T. Namiki, and T. Ito negotiated to provide the placebo and HET with several pharmaceutical companies. M. M., T. Y., T. Nogami, M. A., J. S., K.T., H. N. H. I. and M. N. participated in drafting the work or revising it critically for intellectual content. Y.O., Y.S. and Y.K. participated as statisticians and created a method of randomization. All authors have read and approved the final structured summary.

\section{Authors' information}

T.N. and T. Ito are the vice president and the president, respectively, of the Japan Society of Oriental Medicine (JSOM).

\section{Funding}

This study will be supported in part by funding from the Japan Society of Oriental Medicine. The costs of the ethics review process, office management, statistical analysis, electronic data capture registration, electronic patient-reported outcome registration, data management, and manuscript preparation will be supported by this funding. Ohsugi Pharmaceutical Co. Ltd, Osaka, Japan did not play any part in the design of this study, collection, analysis, and interpretation of data, and in writing the manuscript, other than providing the placebo and HET.

\section{Availability of data and materials}

$$
\text { Not applicable. }
$$

\section{Ethics approval and consent to participate}

Approved by the Ministry of Health, Labour and Welfare (MHLW) Certified Clinical Research Review Board, Chiba University Hospital, Japan, on August 19, 2020 with certificate No. CRB0040-20. The authors certify that this trial has received ethical approval from the appropriate ethical committee as described above.

Before inclusion in the study, conscious patients must be informed of the purpose of the study and of the clinical procedures required by the protocol. The investigators in each hospital will explain the purpose, risks and benefits associated with study participation. In addition, patients will be informed of their right to withdraw from the study at any time without explanation and without losing the right to future medical care.

All participants who recover sufficiently will be given the opportunity to provide their informed consent for ongoing study participation and for the use of data collected for the study. Every patient is free to leave the study protocol at any stage of the study, may withdraw his or her consent, and may request that all of his or her data be eliminated from the database.

\section{Consent for publication}

Not applicable.

\section{Competing interests}

The authors have no competing interests to declare.

\section{Author details}

${ }^{1}$ Department of Japanese-Oriental (Kampo) Medicine, Graduate School of Medicine, Chiba University, 1-8-1, Inohana, Chuo-ku, Chiba 260-8670, Japan. 2Department of Kampo Medicine, Tohoku University Hospital, 1-1, Seiryo-machi, Aoba-ku, Sendai 980-8574, Japan. ${ }^{3}$ Department of Education and Support for Regional Medicine, Tohoku University Hospital, 1- 1, Seiryo-machi, Aoba-ku, Sendai 980-8574, Japan. ${ }^{4}$ Department of Kampo and Integrative Medicine, Tohoku University Graduate School of Medicine, 1-2, Seiryo-machi, Aoba-ku, Sendai 980-8575, Japan. ${ }^{5}$ Community Medicine Education Unit, Graduate School of Medical Science, Kyushu University, 3-1-1
Maidashi, Higashi-ku, Fukuoka 812-8582, Japan. ${ }^{6}$ Department of Pharmacognosy, Graduate School of Pharmaceutical Sciences, Nagoya City University, 3-1 Tanabe-Dori, Mizuho-ku, Nagoya 467-8603, Japan. ${ }^{7}$ Department of Neuropsychiatry, Keio University School of Medicine, 35 Shinanomachi, Shinjuku-ku, Tokyo 160-8582, Japan. ${ }^{8}$ Center for Kampo Medicine, Keio University School of Medicine, 35 Shinanomachi, Shinjuku-ku, Tokyo 160-8582, Japan. ${ }^{9}$ Department of Kampo Medicine, Tokai University School of Medicine, 143 Shimokasuya, Isehara, Kanagawa 259-1193, Japan.

${ }^{10}$ Department of General Medicine/Family \& Community Medicine, Nagoya University Graduate School of Medicine, 65 Tsurumai-cho, Showa-ku, Nagoya 466-8550, Japan. ${ }^{11}$ Department of Traditional Medicine, Faculty of Medicine, Toho University, 5-21-16, Omorinishi, Ota-ku, Tokyo 143-8540, Japan. ${ }^{12}$ Department of Emergency and Critical Care Medicine, Akita University Graduate School of Medicine, 44-2 Hasunuma Hiroomote, Akita 010-8543, Japan. ${ }^{13}$ Department of Infectious Diseases, Chiba University Hospital, School of Medicine, Chiba University, 1-8-1, Inohana, Chuo-ku, Chiba 260-8677, Japan. ${ }^{14}$ Biostatistics Section, Clinical Research Center, Chiba University Hospital, 1-8-1 Inohana, Chuo-ku, Chiba 260-8677, Japan. ${ }^{15}$ Faculty of Nursing, Japanese Red Cross College of Nursing, Tokyo 150-0012, Japan. ${ }^{16}$ Akashi Clinic Kanda, 3-8, Kandaogawamachi, Chiyodaku, Tokyo 101-0052, Japan.

Received: 15 November 2020 Accepted: 30 November 2020 Published online: 06 January 2021

\section{Publisher's Note}

Springer Nature remains neutral with regard to jurisdictional claims in published maps and institutional affiliations.

\section{Ready to submit your research? Choose BMC and benefit from}

- fast, convenient online submission

- thorough peer review by experienced researchers in your field

- rapid publication on acceptance

- support for research data, including large and complex data types

- gold Open Access which fosters wider collaboration and increased citations

- maximum visibility for your research: over $100 \mathrm{M}$ website views per year

At BMC, research is always in progress.

Learn more biomedcentral.com/submissions 